\title{
Electrically switchable photonic liquid crystal devices for routing of a polarized light wave
}

\author{
Irina I. Rushnova ${ }^{a, *}$, Elena A. Melnikova ${ }^{\mathrm{a}}$, Alexei L. Tolstik ${ }^{\mathrm{a}}$, Alexander A. Muravsky ${ }^{\mathrm{b}}$ \\ a Physics Department, Belarusian State University, Nezavisimosti avenue 4, 220030 Minsk, Belarus \\ b Institute of Chemistry of New Materials, National Academy of Sciences, Fr. Skaryna Street 36, 220141 Minsk, Belarus
}

\section{A $\quad$ R}

\section{Keywords:}

Beam splitters

Liquid crystal devices

Optical device fabrication

Optical switches

Patterned photoalignment

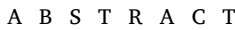

The new mode of LC alignment based on photoalignment AtA-2 azo dye where the refractive interface between orthogonal orientations of the LC director exists without voltage and disappeared or changed with critical voltage has been proposed. The technology to fabricate electrically controlled liquid crystal elements for spatial separation and switching of linearly polarized light beams on the basis of the total internal reflection effect has been significantly improved. Its distinctive feature is the application of a composite alignment material comprising two sublayers of Nylon- 6 and AtA-2 photoalignment azo dye offering patterned liquid crystal director orientation with high alignment quality value $q=0.998$. The fabricated electrically controlled spatially structured liquid crystal devices enable implementation of propagation directions separation for orthogonally polarized light beams and their switching with minimal crosstalk.
\end{abstract}

(C) 2017 Elsevier B.V. All rights reserved.

\section{Introduction}

At the present time the integrated optics technologies are actively developed because they enable designing of innovative functional elements to control the spatial and polarization characteristics of radiation being compact, reliable, and efficiently produced [1,2]. Among the materials for the creation of such devices, liquid crystals (LC) are very promising due to their high birefringence $(\Delta n \sim 0.1-0.5)$ and due to the possibility for its control by low applied voltages $(1-10 \mathrm{~V})[3,4]$. With the use of liquid crystals, various imaging systems [5], optical switches [6,7], spatial light modulators [8], diffractive elements [9] are manufactured; LC integrated components having sophisticated optical anisotropy are developed for fiber optical communication lines and for the data processing and communication devices (compact optoelectronic waveguides, dividers, multiplexers, etc.) [10]. The operation principle of these LC devices is based on the effect of refraction and reflection of a light wave propagating within the LC layer, at the interface of two LC regions with different orientations of the director [11].

Analysis of different combinations for mutually orthogonal orientations of the LC director in the adjacent domains of a nematic liquid crystal has made it possible to find the optimum configurations for realization of the total internal reflection (TIR) effect of linearly polarized light beams [12]. Using a planar orientation of the director in the perpendicular directions, and also the planar and homeotropic orientations of the director in adjacent regions of the element, spatial separation of $s$ - and $p$-polarization of the light beams at the domains interface is realized.

It should be noted that the early attempts of such LC elements manufacturing $[12,13]$ were successful at realization of the polarization separation effect but failed to ensure a low level of crosstalk. Besides, a thickness of an LC layer in the manufactured LC elements was about $200 \mu \mathrm{m}$ and required the use of high control voltages (50-100 V).

This paper presents a technological innovation to produce LC elements with the electrically controlled interface between two LC domains with orthogonal orientations of the director. Three options of director orientation in LC cells have been chosen to provide reflection of one polarized radiation component and full (almost full) transmission of the orthogonally polarized component as well as their switching when the reflected and transmitted beams interchange their positions under the effect of an applied electric field.

\section{LC cells and their fabrication technology}

To realize the total internal reflection effect, we select two geometries associated with a planar orthogonal orientation of the director in two regions of the element (Fig. 1(a)) and with the planar and homeotropic orientations of the director (Fig. 1(b)). As noted, such

\footnotetext{
* Corresponding author.

E-mail address: Rushnova@bsu.by (I.I. Rushnova).
} 

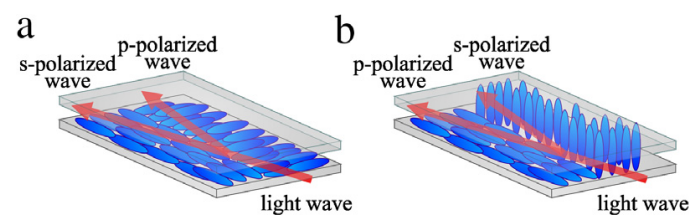

Fig. 1. Options of the LC director orientations: (a) - planar orthogonal orientation of the director in adjacent regions; (b) - planar and homeotropic orientations of the director in adjacent regions.
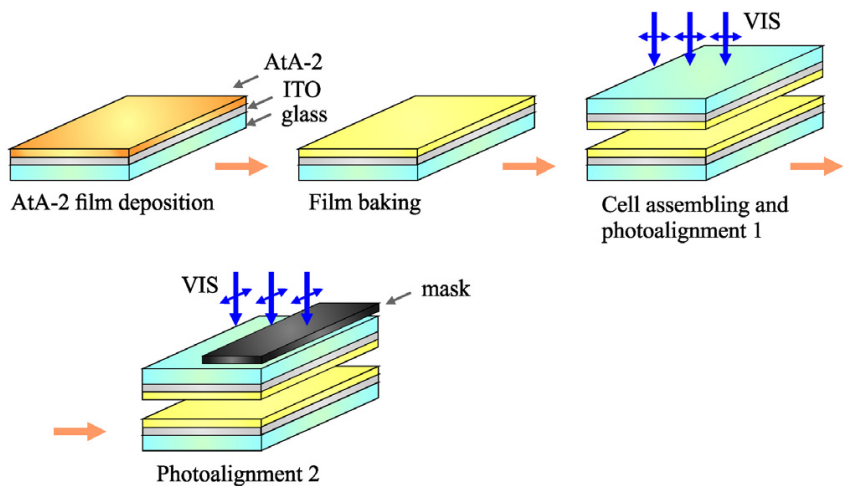

Fig. 2. Process flow of the LC cell fabrication with two domains planar photoalignment of LC.

options of the director orientation enable design of LC devices for separation of the polarized light beams [13].

The initial molecular orientation of a nematic LC was set by the contactless photoalignment method [14,15]. The AtA-2 azo dye [16] synthesized at the Institute of Chemistry of New Materials of the Belarusian National Academy of Sciences, that is characterized by high azimuthal anchoring energy $\left(>1 \times 10^{-4} \mathrm{~J} / \mathrm{m}^{2}\right)$, thermostability and photostability [17], was selected as an alignment material. LC photoalignment direction of AtA-2 film is efficiently induced by linearly polarized 440-460 nm LED light. Opportunity of visible light utilization for photoalignment offers exposure of the assembled LC cells with glass substrates on both sides, thus simplifying fabrication and reducing the price of photonic LC devices.

The planar orthogonal orientation of the director in both regions of LC cells was created by the two stage photoalignment method illustrated in Fig. 2.

The AtA-2 alignment layer was wet deposited on glass substrate with transparent conducting layer (ITO) from $2 \%$ 2,2'-Dimethylformamide solution using rod coater. The sample was baked at $T \sim 100-140{ }^{\circ} \mathrm{C}$ to remove the residual solvent. The reversibility of AtA-2 photoalignment makes it possible to change locally and to modify multiple times the previously formed direction of LC orientation. This feature forms the basis for the formation of planar orthogonal LC director domains. First, assembled cell is exposed with linearly polarized LED light with intensity $I=15 \mathrm{~mW} / \mathrm{cm}^{2}$ during the period $t=20 \mathrm{~s}$. Then the electrical vector direction is rotated by $90^{\circ}$ and the cell is exposed again, through the amplitude mask that covers half of the sample, for $t=60 \mathrm{~s}$. Molecular orientation of the azo dye in the region of the cell covered by the mask is invariable, as distinct from the adjacent region, where AtA-2 layer forms new LC alignment direction perpendicular to the electrical vector of linearly polarized light exposure.

The alignment quality of the LC molecular orientation in the photoaligned samples was evaluated by the follow expression:

$q=\frac{T_{\max }-T_{\min }}{T_{\max }+T_{\min }}$,

where $T_{\max }-$ maximal (in the case of parallel polarizers) and $T_{\min }-$ minimal (in the case of crossed polarizers) transmittance of the sample.
Measured in this way quality parameter $q$ for the manufactured samples is $q=0.996-0.998$ at the wavelength $\lambda=632.8 \mathrm{~nm}$.

In the LC cell shown in Fig. 1(b), the refractive index interface is formed due to the applied electric field. Using structured electrode at one of the cell substrates, one can set a homeotropic orientation of the director in the region of the electrode by application of electric field higher than the threshold value [18]. In the other part of the cell, the initial planar LC director orientation is maintained. To create a conducting layer covering a part of the cell, as a rule, the photolithographic method is used [19]. The difficulty in manufacturing of such LC devices is associated with the fact that, in the process of the pattern formation at the conducting layer, it loses homogeneity, at the boundary with the etched electrode some spikes are formed hindering deposition of a uniform thin film (20-100 nm) of the photoalignment AtA-2. In cells with the partial conducting layer, the orientation quality parameter $q$ is usually below $q \leq 0.930$ causing a high level of crosstalk when the light beams are switched over.

To improve the alignment quality of LC cell with patterned photoalignment the original technology is implemented based on a composite photoalignment material that combines Nylon- 6 and photoalignment AtA-2 azo dye. Adding buffer layer (Nylon-6) allows improvement of photoalignment layer characteristics [20]. Fig. 3 demonstrates the manufacturing process flow of LC cells with the planar and homeotropic orientations of the director.

A glass plate with an opaque conducting layer of chromium is used as a lower substrate. First, the chromium layer is partially etched from a half of the substrate by the photolithographic method. Next Nylon6 sublayer is uniformly deposited on top the substrate and baked at $180^{\circ} \mathrm{C}$ for $1 \mathrm{~h}$. The Nylon- 6 smooths the spikes formed at the interface of etched chromium and improves the sample surface. The uniform thin film of AtA- 2 azo dye is deposited on top of Nylon- 6 sublayer and baked at $T \sim 100-140{ }^{\circ} \mathrm{C}$ for $5 \mathrm{~min}$. The upper substrate is manufactured by the standard technology when the photoalignment material is deposited to a glass plate with a transparent conducting layer and then the sample is baking.

Assembled cell is exposed with linearly polarized LED light with intensity $I=15 \mathrm{~mW} / \mathrm{cm}^{2}$ during the period $t=20 \mathrm{~s}$ to obtain a homogeneous planar orientation of LC molecules. The orientation quality parameter of the manufactured samples comes to $q=0.996-0.998$ at the wavelength $\lambda=632.8 \mathrm{~nm}$ being much higher, when the Nylon- 6 layer was not used. A thickness of the LC layer in the manufactured elements is about $20 \mu \mathrm{m}$, enabling the control over the refractive interface at the voltages up to $5 \mathrm{~V}$. The cells were filled with nematic liquid crystal LC-1289 (developed at the Research Institute of Organic Intermediate Products and Dyes, Moscow) with the refractive index anisotropy $\Delta n=$ 0.156 at the wavelength $\lambda=632.8 \mathrm{~nm}\left(n_{e}=1.69, n_{o}=1.53\right.$ for all cases of alignment materials in presented technologies). The order parameter $\mathrm{S}$ of the LC-1289 obtained from the multiple frustrated total internal reflection method of IR spectroscopy is $S=067$ [21]. The threshold voltage for realignment of the LC director is $U_{t r}=1 \mathrm{~V}$.

\section{Experimental results and discussion}

Linearly polarized radiation of a helium-neon laser with the wavelength $\lambda=632.8 \mathrm{~nm}$ and the power $P=300 \mu \mathrm{W}$, focused by the 10 lens (waist diameter $d=16 \mu \mathrm{m}$ ), was input into the end of LC cells at the incidence angle exceeding the critical angle for the TIR [22]. Table 1 presents experimental photographs of the laser radiation propagation in LC cells depending on polarization of the incident radiation and on the voltage applied to the cell. Indicated in Table 1 angle $\alpha$ between the normal of the interface and the light beam differs from the incidence angle $2^{\circ}$ only for set directions of LC orientation. The deviation of the incident angle from the light beam derives from the theoretical issue of the relation between wavenumber vector and Poynting vector in the anisotropic media [22]. It should be noted, the angular difference between the wavenumber vector and the Poynting vector is much less 
Table 1

Propagation of the linearly polarized light beams in the electrically controlled LC cells.

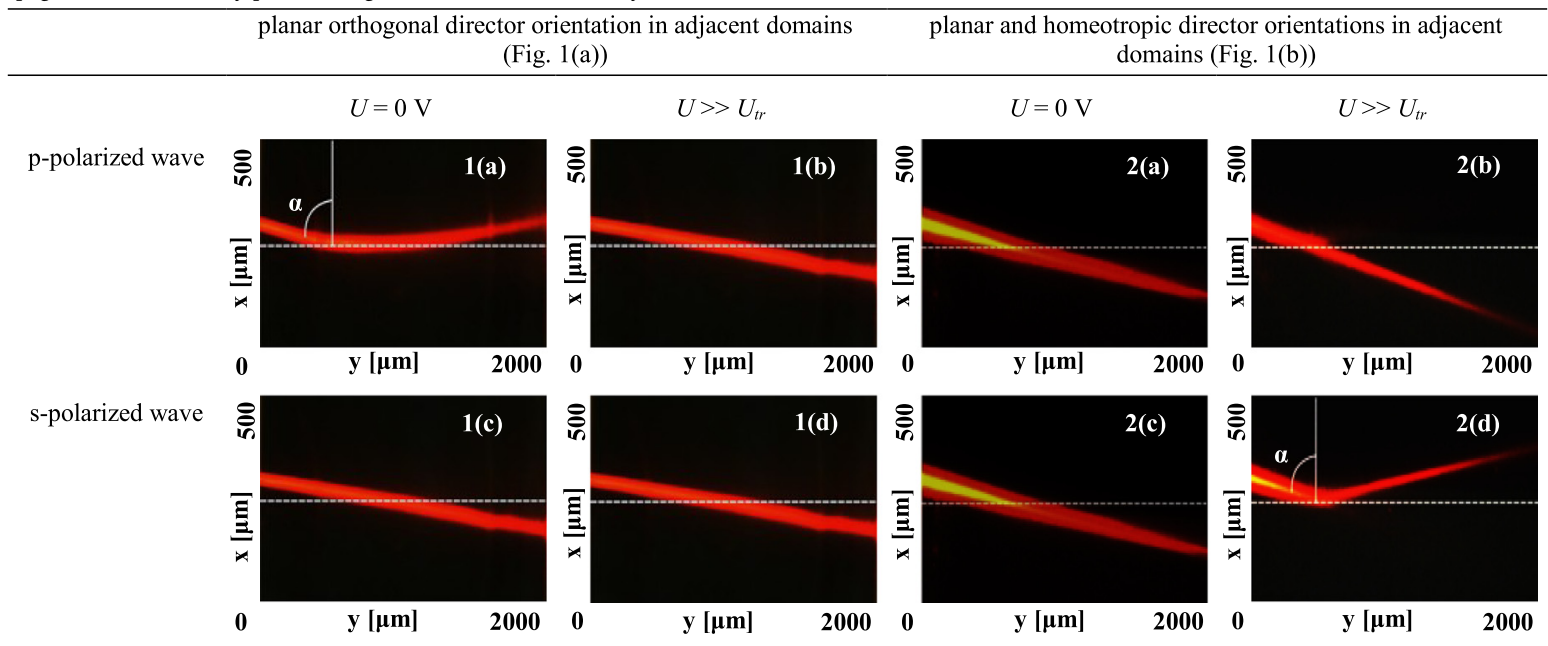
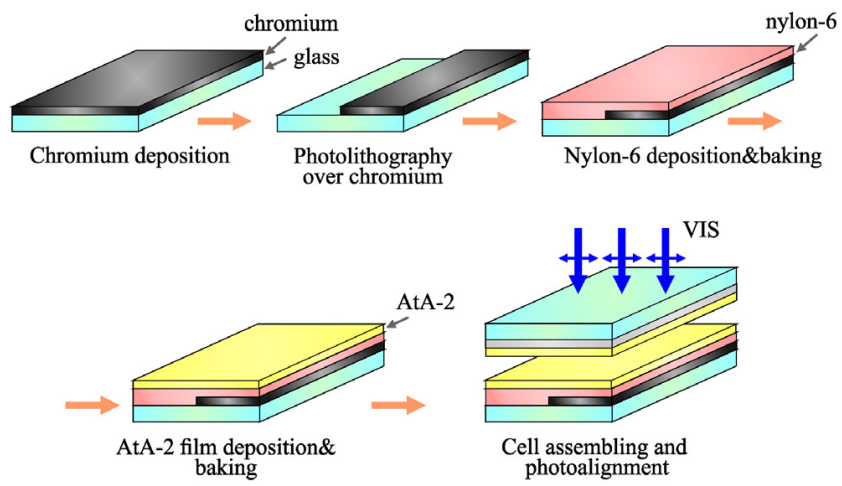

Fig. 3. Process flow of fabrication of the LC cells with structured electrode and planarhomeotropic orientations of the director in two regions of the element.

than the difference between the incidence angle $80^{\circ}-82^{\circ}$ and the TIR angle which is below $70^{\circ}$.

As seen, in the LC cell with a planar orthogonal orientation of the director in the absence of the applied electric field, for a wave with $p$-polarization the TIR at the interface (white dashed line) of two orientations of the LC director (Table 1, 1(a)) is fulfilled. And the $s$-polarized wave passes the interface without any changes in its propagation direction (Table $1,1(\mathrm{c})$ ).

Indeed, for a wave with $s$-polarization, in both regions of the cell the directions of the electric field vector $\mathbf{E}$ and of the LC director $\mathbf{n}$ are perpendicular, both regions being characterized by the ordinary refractive index $n_{o}$. For a wave with $p$-polarization, in the beam incidence region (where the director $\mathbf{n}$ is parallel to the LC cell end) the electric field vector $\mathbf{E}$ and the LC director $\mathbf{n}$ make an angle other than $0^{\circ}$ and $90^{\circ}$ and hence the region is determined by the effective index of refraction $n_{e f 1}$ that is given by

$n_{e f 1}(\theta)=\frac{n_{o} n_{e}}{\sqrt{n_{o}^{2} \sin ^{2} \theta+n_{e}^{2} \cos ^{2} \theta}}$,

where $\theta$ - incidence angle; $n_{o}$ and $n_{e}$ - ordinary and extraordinary refractive indices.

The adjacent region is determined by the effective index of refraction $n_{e f 2}$ according to the formula

$n_{e f 2}(\theta)=\frac{n_{o} n_{e}}{\sqrt{n_{o}^{2} \cos ^{2} \theta+n_{e}^{2} \sin ^{2} \theta}}$.
When the incidence angles are $\theta>45^{\circ}$, the region with the effective index of refraction $n_{e f 1}$ is optically denser that the region with $n_{e f 2}$, enabling realization of TIR for $p$-polarized radiation at the angles greater than the critical.

When voltage is applied to the LC cell, LC molecules are reoriented (Fredericks transition [18]), a homogeneous homeotropic orientation of the LC director is created within the whole volume of the LC cell, and light beams propagate rectilinearly regardless polarization (Table 1 , 1(b), 1(d)).

In an LC cell with the electrically induced interface for $U \gg U_{t r}$, a wave with $s$-polarization is subjected to the TIR as it illustrated in Table 1, 2(d), whereas a wave with $p$-polarization passes the interface being refracted as it shown in Table 1, 2(b). Really, for the s-polarized radiation, in the region of incidence (from the side of the opaque electrode made of chromium) an extraordinary wave is excited with the refractive index $n_{e}$ due to identical directions of the electric field vector $\mathbf{E}$ and the director $\mathbf{n}$. In the adjacent region an ordinary wave is excited with the refractive index $n_{o}$. So, a light wave propagating from the region with a higher refractive index to that with the lower refractive index is subjected to TIR when incident on the interface at the angle that is greater than the critical angle (Table 1, 2(d)).

Electromagnetic wave with $p$-polarization $\left(U \gg\left(U_{t r}\right)\right.$ in the region of the beam incidence is characterized by the ordinary refractive index $n_{o}$, and in the adjacent region - by the effective index of refraction given by formula (3). Propagating from the region with a lower refractive index $n_{o}$ to the region with a higher one $n_{e f 2}$, the $p$-polarized light wave at the interface is divided into the reflected and refracted waves. In the selected director orientation geometry, according to the Fresnel formulas, the reflection factor is below $0.2 \%$ and is responsible for low intensity for the reflected wave (Table 1, 2(b)). It should be noted, for incident angle $\theta=80^{\circ}$ an angular deflection of the refracted beam is $0.9^{\circ}$ only.

In the absence of applied electric field, the molecular orientation in such LC cell is homogeneous and planar, the beams propagate rectilinearly regardless polarization as it illustrated in Table 1, 2(a), 2(c).

On excitation of the waves with $p$-polarization or with $s$-polarization, light within the LC layer is either reflected (Table 1, 1(a), 2(d)) or refracted (transmitted) (Table 1, 1(b)-(d), 2(a)-(c)), and the Contrast Ratio $\left(C R=T_{\max } / T_{\min }\right)$ of the elements under study is $C R>100: 1$, being much higher than in the previous works [12,13], where $C R$ was never higher than 10:1.

Propagation of light beams in the spatially structured LC cells has been studied on smooth rotation of polarization vector, with the use of the half wave plate $\lambda / 2$, at the invariable light beam intensity. The direction of oscillations for the electric vector $\mathbf{E}$ of a light wave is taken counterclockwise from the axis $O X$, when the angle $\beta=0^{\circ}$ is associated 
Table 2

Spatial polarization separation of linearly polarized laser radiation at the interface of two regions.

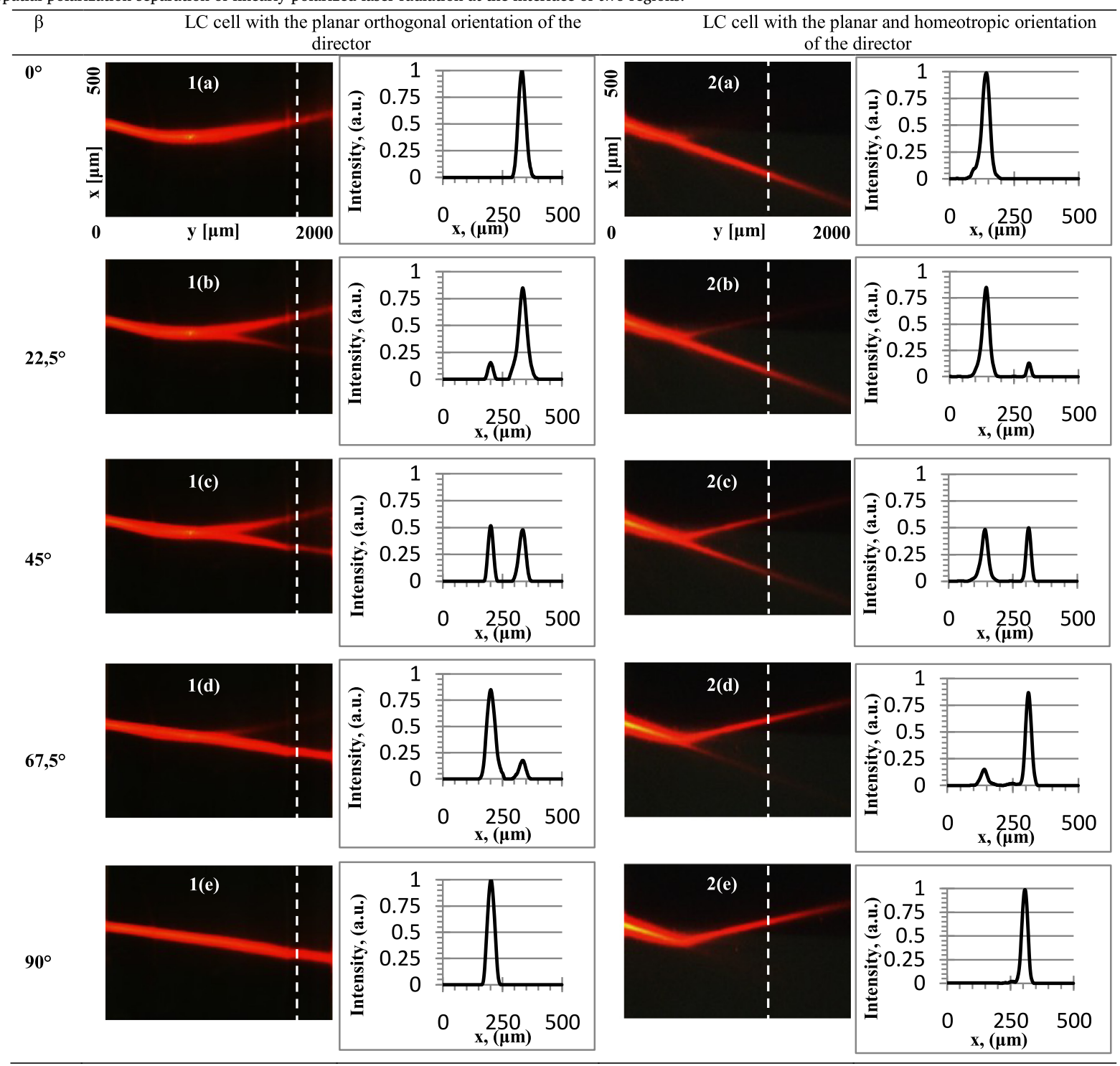

with the $p$-polarized wave, whereas the angle $\beta=90^{\circ}$ corresponds to $s$-polarization. Table 2 presents experimental photographs of the radiation propagation within LC cells when using a helium-neon laser and the intensity distribution graphs in the cross-section indicated by a white dashed line. It is seen that a smooth variation of the light wave polarization vector direction offers the spatial polarization separation of a light beam. As expected, for example, for the angle $\beta=45^{\circ}$, both polarization components are of the identical intensity. It was checked experimentally that the polarization of light that passed through the LC layer was preserved in set directions of LC orientation.

Based on a quantitative analysis of the cross-sectional intensity distribution, one can obtain the curves for intensity of the transmitted (refracted) and reflected beams as a function of the polarization vector rotation angle as it shown in Fig. 4 for two options of the director orientation in the adjacent domains. The experimental results are in a good agreement with the theoretical calculations illustrated in Fig. 4 by solid lines. The calculations have been performed for separation of the wave into two components associated with $s-\left(A_{s}\right)$ and $p$-polarizations $\left(A_{p}\right)$ :

$A_{s}=A_{0} \sin \beta$,

$A_{p}=A_{0} \cos \beta$, where $A_{0}$ - amplitude of the incident wave, $\beta-$ angle between the direction of oscillations for the electric vector $\mathbf{E}$ and the axis $O X$.

Combining the two geometries for the director orientation shown in Fig. 1 in a one LC device, one can realize not only spatial separation of the orthogonally polarized light beams but also their switching when the reflected and transmitted light beams interchange their positions under the action of the applied electric field [19]. The use of the lower substrate with a partially opaque conducting layer of chromium and setting of the initial planar orthogonal orientation enables one to realize reflection of a wave with $p$-polarization. In this case the interface between two orientations is narrow $(\approx 4 \mu \mathrm{m})$ (Fig. 5(a)) due to the proposed modified technology of LC photoalignment, offering strong anchoring energy for small width of the boundary disclination. When an electric field is applied to such an LC element, in the region with chromium the molecular orientation becomes homeotropic to provide the conditions for reflection already of the s-polarized wave. Fig. 5(b), (c) show the photographs for the LC element operating without and with the electric field applied. In the process of work a $p$-polarized light beam of a helium-neon laser $(\lambda=632.8 \mathrm{~nm})$ and a $s$-polarized second-harmonic beam of a diode-pumped neodymium doped yttrium aluminate garnet laser $(N d: Y A G)(\lambda=532 \mathrm{~nm})$ were used. Both beams were input into the end of the electrically controlled LC element from the 

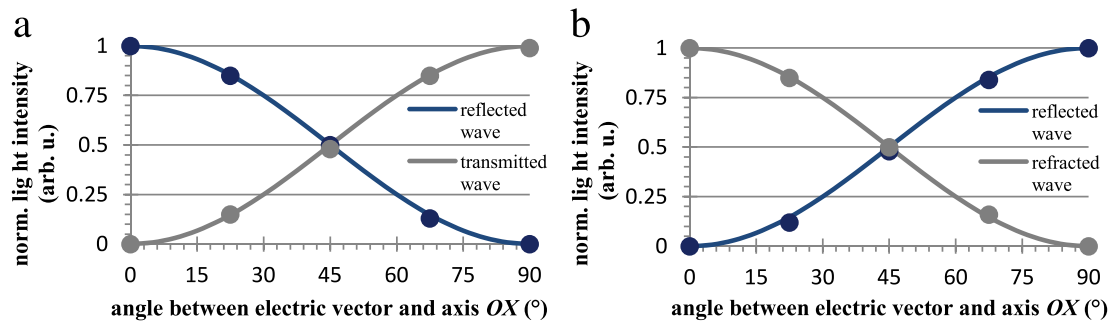

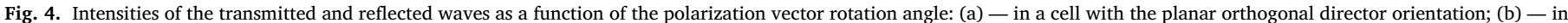
a cell with the electrically induced interface of the director orientation.
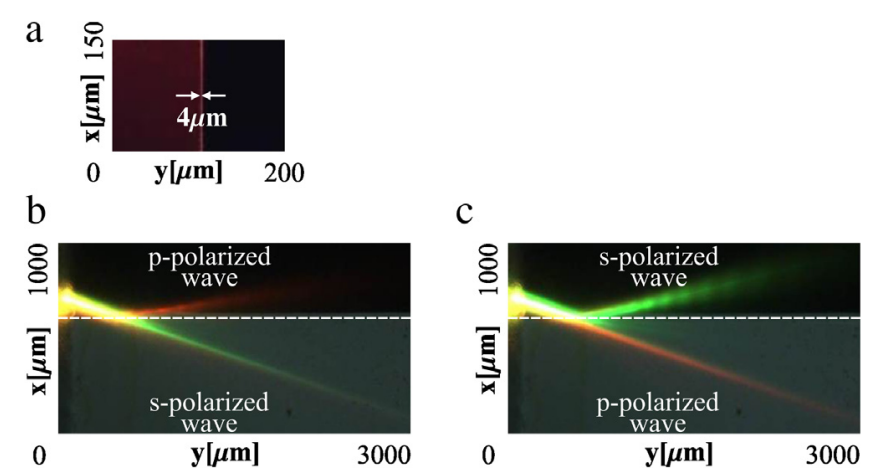

Fig. 5. (a) - Experimental photograph of the interface between two orthogonal LC director orientations for the crossed polarizers; (b), (c) - switching effect of the orthogonally polarized light beams in the electrically controlled LC cell with the refractive interface ( $p$-polarized - radiation of a He-Ne laser, $s$-polarized — radiation of a Nd:YAG laser) for $U=0$ (b) and $U=3.5 \mathrm{~V}$ (c). (For interpretation of the references to color in this figure legend, the reader is referred to the web version of this article.)

side of the initial director orientation parallel to the end. The incidence angle $\theta=75^{\circ}$ was chosen above the critical one.

As seen from the obtained experimental data, without applied voltage, the $p$-polarized wave (red) is subjected to the TIR, whereas the $s$ polarized wave (green) is transmitted through the interface without any changes in its propagation direction (Fig. 5(b)). When the applied voltage exceeding the threshold value $\left(U \gg\left(U_{t r}\right)\right.$, the total internal reflection condition is already observed for the $s$-polarized wave (Fig. 5(c)). When two orthogonally polarized waves ( $s$ - and $p$-polarization) are propagating simultaneously, one can observe polarization separation of the radiation at the interface, the light beams interchange their positions under the action of the applied electric field.

\section{Conclusion}

This work presents a new technology for photoalignment of liquid crystal cells based on a composite material that combines Nylon- 6 and AtA-2 azo dye sublayers. Owing to the two stage photoalignment method, the electrically controlled LC elements with the interface between two domains characterized by orthogonal LC director orientations with alignment quality parameter $q=0.998$ can be designed. Based on this technology, the electrically controlled LC devices providing efficient separation and low crosstalk switching of propagation directions of the orthogonally polarized light waves have been successfully fabricated.

\section{Acknowledgments}

This work was supported by the Belarusian State Program of Scientific Investigations "Convergence 2016-2020" No. 3.03.5.
The authors express their gratitude to Dr. V.S. Mikulich from the Materials and Technologies of LC Devices Laboratory (Institute of Chemistry of New Materials, National Academy of Sciences) for the opportunity to use the material AtA-2 in the process of work.

\section{References}

[1] H.-S. Kwok, S. Naemura, H.L. Ong, Progress in Liquid Crystal Science and Technology, Vol. 4, World Scientific Publishing Company, Singapore, 2013.

[2] V.G. Chigrinov, Liquid Crystal Photonics: Engineering Tools, Techniques and Tables, Nova Science Publishers, New York, 2014.

[3] A.G. Maksimochkin, S.V. Pasechnik, G.I. Maksimochkin, V.G. Chigrinov, Electrically controlled waveguide mode in LC layer for fiber optic applications, Opt. Commun. 283 (2010) 3136-3141.

[4] J. Beeckman, K. Neyts, P. Vanbrabant, Liquid-crystal photonic applications, Opt. Eng. 50 (2011) 08120201-08120217.

[5] R.H. Chen, Liquid Crystal Displays: Fundamental Physics and Technology, first ed., Wiley, New Jersey, 2011.

[6] A.G. Maksimochkin, S.V. Pasechnik, V.A. Tsvetkov, D.A. Yakovlev, G.I. Maksimoch kin, V.G. Chigrinov, Electrically controlled switching of light beams in the plane of liquid crystal layer, Opt. Commun. 270 (2007) 273-279.

[7] R. Barboza, A. Alberucci, G. Assanto, Electro-optic beam steering with nematicons, Mol. Cryst. Liq. Cryst. 558 (2012) 12-21.

[8] J.P. Parry, R.J. Beck, J.D. Shephard, D.P. Hand, Application of a liquid crystal spatial light modulator to laser marking, Appl. Opt. 50 (2011) 1779-1785.

[9] A.A. Kazak, A.L. Tolstik, E.A. Melnikova, Controlling light fields by means of liquidcrystal diffraction elements, J. Opt. Technol. 77 (2010) 461-462.

[10] O.S. Kabanova, E.A. Melnikova, I.I. Olenskaya, A.L. Tolstik, Electrically controlled waveguide liquid-crystal elements, Tech. Phys. Lett. 40 (2014) 598-600.

[11] M. Peccianti, A. Dyadyusha, M. Kaczmarek, G. Assanto, Tunable refraction and reflection of self-confined light beams, Nat. Phys. 2 (2006) 737-742.

[12] A. Komar, A. Tolstik, E. Melnikova, A. Muravsky, Optical switch based on the electrically controlled liquid crystal interface, Appl. Opt. 54 (2015) 5130-5135.

[13] A.A. Komar, M.A. Kurochkina, A.A. Melnikova, A.I. Stankevich, A.L. Tolstik, Polarization separation of light beams at the interface of two mesophases, Tech. Phys. Lett. 37 (2011) 704-706.

[14] V. Chigrinov, H.-S. Kwok, H. Hasebe, H. Takatsu, H. Takada, Liquid-crystal photoaligning by azo dyes, J. Soc. Inf. Disp. 16 (2008) 897-904.

[15] A. Muravsky, Next Generation of Photoalignment, VDM Verlag Dr. Müller, Saarbrücken, 2009.

[16] V.S. Mikulich, An.A. Murawski, Al.A. Muravsky, V.E. Agabekov, Influence of methyl substituents on azo-dye photoalignment in thin films, J. Appl. Spectrosc. 83 (2016) $115-120$.

[17] Al. Muravsky, V. Mikulich, An. Murauski, V. Bezruchenko, New photoalignment material: azimuthal anchoring energy decreases at very high photo-induced order parameters, SID Symp. Dig. Tech. Pap. 115 (2015) 1602-1604.

[18] L.M. Blinov, Structure and Properties of Liquid Crystals, Springer, 2011.

[19] E.A. Melnikova, A.L. Tolstik, I.I. Rushnova, O.S. Kabanova, A.A. Muravsky, Electrically controlled spatial-polarization switch based on patterned photoalignment of nematic liquid crystals, Appl. Opt. 55 (2016) 6491-6495.

[20] I. Rushnova, An. Murauski, V. Mikulich, Al. Muravsky, High anchoring composite photoalignment material with high photosensitivity, in: The 23th international Display Workshops (IDW/AD), 2016, pp. 69-72.

[21] E.A. Konshina, E.O. Gavrish, A.I. Vagnonen, Opt. Spectr. 120 (2016) 250-254.

[22] E. Hecht, Optics, Addison Wesley, 2002 